\title{
Volatile inhibitors of atmospheric corrosion. III. Principles and methods of efficiency estimation
}

\author{
N. N. Andreev* and Yu. I. Kuznetsov \\ A. N. Frumkin Institute of Physical Chemistry and Electrochemistry, Russian Academy \\ of Sciences, Leninskii pr. 31, Moscow, 119071 Russian Federation \\ *E-mail: n.andreev@mail.ru
}

\begin{abstract}
This paper continues the analytical literature review on the theory and practice of metal protection by volatile corrosion inhibitors (VCI) supplemented by our own data. The principles and methods for fast estimation of VCI efficiency are reviewed.
\end{abstract}

Key words: volatile corrosion inhibitors, efficiency estimation, accelerates tests.

Received: February 1, 2013

doi: $10.17675 / 2305-6894-2013-2-1-039-052$

\section{Introduction}

Selection of VCIs (or materials capable of vapor-phase protection of metals) for specific purposes and optimization of product composition during development require that their anti-corrosion properties be estimated [1]. The estimation is based on both laboratory tests and field tests [2].

Field tests under real conditions provide the most accurate results. We believe that it is a mandatory step in large-scale application of VCI-based preservative agents and the decisive basis in material selection. However, such tests are time consuming, do not provide fast feedback to a researcher, and are often laborious and expensive.

Laboratory tests can be classified into simulation tests and accelerated tests. The former reproduce typical conditions encountered during transportation, storage, or operation of items being protected. If the test modes are chosen correctly, these tests have better reproducibility than field tests but are generally as time consuming. Therefore, the use of accelerated methods to study the protective effects of VCIs, reject products with poor potential, and/or determine ways for modifying such products is a necessary stage that precedes field tests.

The diversity of tasks solved by VCIs, the forms and methods of their application, and the complexity of vapor-phase protection mechanisms do not allow accelerated tests to be unified completely. This incited the development of numerous methods [2-7], some of which are quite specific. Attempts at generalization and formal transfer of their results to systems different from those being simulated often result in a non-optimal choice of materials. They resulted in the opinion that accelerated tests cannot reflect the real properties of VCIs. 
One can agree with the opinion [8] about the complexity of predicting the real longevity of metal protection by VCIs accurately based on accelerated test results. However, the statement that they, at best, only provide information about the principal suitability of a product for vapor-phase protection appears too pessimistic.

The purpose of this overview was to analyze:

- approaches for fast estimation of efficiency of VCIs and materials on their basis;

- factors affecting the validity of results of such estimation;

- informativity of the most popular accelerated test methods;

- prospects of developing accelerated methods for testing the efficiency of temporary protection means of this type.

\section{General principles}

Accelerated tests of VCIs can be arbitrarily classified into "indoor tests" and "cabinet tests".

"Indoor methods" are most popular and diverse. The simplicity of instrumentation used is their obvious advantage [2]. They rarely reproduce a real system being protected. However, they allow one to judge on the feasibility of using a compound as a VCI "in general", can simulate the reasons of vapor-phase protection failure under various conditions, and help one to estimate, sometimes "in general", some characteristics of VCIs affecting their functional properties.

"Cabinet" tests imply that special cabinets are used that can vary the temperature, humidity, and other environment characteristics according to a certain program. They can simulate the most drastic conditions of storage and operation of a real item (from corrosion perspective), or create conditions that are even more difficult for protection and accelerate the experiment even more.

It is believed that in some cases cabinet tests can estimate the real protection times of particular items with some accuracy [4]. However, reliable coefficients for conversion of accelerated test results for transportation, storage, or operation conditions of VCI-protected items are not available, so protective properties of VCIs are usually predicted on the basis of comparative data. The prediction logic is the same for "cabinet" and "indoor" tests: if a product or material being tested demonstrates a higher efficiency than some other product or material in accelerated tests, it can be expected that it will also be more efficient under natural conditions.

The validity of such predictions depends on how precisely analysis of a real system can single out the factors that violate the protection of the item and reproduce these factors in the tests $[2,9]$. However, even if the systems are ideally similar, a minor scatter of results provided by express methods may produce critical inaccuracies in long-term prediction of VCI efficiency. Obviously, the risk of errors grows with the ratio of the forecast time to the test time, hence "too fast" tests should generally be avoided.

In many cases, additional errors in the estimation of VCI functional properties are introduced if special metal specimens are used in tests. This approach is justified for 
optimizing the characteristics of temporary protection means during development. However, if a material is selected for a particular practical purpose, it is desirable to perform the tests with the items to be protected if the characteristics of these items permit. The corrosion behavior of assembled "multi-metal" items is hard to simulate in laboratory. What is more, it is preferable to perform experiments with items packed nearly in the same manner as they are actually packed.

Choosing the estimation criterion is an important issue in VCI testing. In practice, corrosion protection of an item is understood as hindering corrosion to a level that ensures the preservation of the operational and/or decorative properties of the item. Since VCIs are often used for the preservation of high-precision items that can be made inoperative even by a minor damage, corrosion prevention is the most common feature of vapor-phase protection of a system $[1,2]$. Thus, unless anything else is required for a particular task, characteristics reflecting conditions of complete metal protection should be used, such as the VCI dosage ensuring protection [10], time until the first corrosion damage [2], protection radius $[3,11,12]$, etc. At the same time, characteristics such as corrosion rates, inhibition factors, degrees of metal protection, areas of corrosion damage, and similar characteristics should be considered as useful but secondary.

Having determined the criteria of metal protection by VCIs, let us briefly consider the processes and factors that result in protection failure.

According to [13-16], protection of a system is primarily determined by the initial period of metal exposure in a limited volume containing a VCI. If inhibitor vapors diffusing from a source create a VCI concentration preventing corrosion in the surface moisture film before a corrosion site appears on the surface, the system will be protected from corrosion for a long time if no interactions with the environment occur. In practice, if the inhibitor volatility and ability to prevent corrosion in the surface electrolyte are insufficient, and hence the protection radius is small, corrosion sites on the item appear almost at once after the preservative packing. This can often be avoided by performing preservation under mild conditions (from the corrosion point of view), keeping the system under such conditions after the preservation, and uniform distribution of VCI sources in the volume being protected.

Mass exchange of a system with the environment, such as VCI evaporation from it or inward diffusion of corrosive environment components, including water, can result in a decrease in the inhibitor concentration in the system, an increase in the corrosivity of the inside atmosphere in the space being protected, and hence failure of vapor-phase protection [13-16].

An increase in environment corrosivity and in inhibitor protective concentration upon variation of temperature can result in corrosion initiation even in perfectly sealed systems protected with a VCI. Furthermore, heat exchange processes cause VCI redistribution in the electrolyte on the metal, similarly to that occurring during distillation of solutions. If the 
surface concentration of the inhibitor decreases below the protective level, vapor-phase protection also fails [13-19].

It is the mass exchange and heat exchange processes that most commonly determine the duration of vapor-phase protection for real objects.

\section{Indoor methods}

"Indoor tests" can be separated into liquid-phase, gas-phase, and mixed ones.

\subsection{Liquid-phase tests}

Liquid-phase tests allow one to make the most reliable conclusions on the functional properties of materials and chemicals application of which implies contact or proximate location of a VCI carrier with a metal, such as preservative solutions, oils, and process liquids, packing papers and films, etc. The factors accelerating corrosion in this group of tests include the mode of metal interaction with the electrolyte and the corrosive additives introduced. The materials to be tested are initially placed in model electrolytes such as distilled water $[4,10]$ or salt solutions [1]. The protective capability of VCIs can be studied in the bulk of such electrolytes or in their sufficiently thick films by corrosion or electrochemical methods.

Tests in bulk electrolytes simulate atmospheric corrosion conditions less thoroughly than tests under films of electrolytes. However, the simplicity of the former and the similarity of changes in the protective properties of inhibitors in bulk and surface solutions [20] justify their widespread use. Both electrochemical and corrosion tests of this type are similar to those used to estimate the properties of other classes of water-soluble inhibitors, such as potentio- and galvanostatic and -dynamic methods, chronopotentiometry, gravimetry, etc.

The methods for the study of the electrochemical behavior of metals in electrolyte films [2], including those adapted to estimate the protective effect of inhibitors, did not become popular in VCI testing because rather complex instrumentation is required. Of corrosion methods, the so-called "blade" test [7] is likely to be the most popular. During this test, a drop of water or salt solution is placed on a metal and covered with a piece of VCIcontaining paper or film. According to [7], the corrosion state of the metal surface after a certain time is estimated by visual examination and compared to the state of a reference specimen covered by a similar material without an inhibitor. On the other hand, the minimum content of the VCI in the material that ensures corrosion prevention or other parameters can also be used as efficiency criteria in this test.

Liquid-phase tests provide information that is useful for VCI development and optimization of VCI properties. Other conditions being equal, a VCI is the more efficient the higher its inhibiting effect in an electrolyte simulating the surface electrolyte. However, in the case of vapor-phase protection, this information is significant only if the inhibitor is 
sufficiently volatile. ${ }^{1}$ In the absence of experimental or calculated vapor pressure data, the tests described above do not even allow conclusions to be made on whether an inhibitor is principally suitable as a VCI. This is achieved by gas-phase tests in which the metal, VCI source, and corrosive electrolyte are spatially separated, and VCI transportation to the surface being protected involves vaporization.

\subsection{Gas-phase tests}

In these tests, the accelerating factors can include elevated humidity, corrosive additives, and temperature conditions that are unfavorable for vapor-phase protection. A VCI and an aqueous electrolyte are placed in a cell, either as a VCI solution in the electrolyte or separately. In the latter case, a test is generally preceded by exposing the metal to VCI vapors in an environment with reduced corrosivity, which simulates the conditions of item preservation and is required for accumulation of the inhibitor on the surface, its adsorption, and formation of protective layers.

Volatile acids, acid gases, etc. can be introduced into the test cell [7]. On the one hand, the choice of the corrosive additive in gas-phase tests depends on the specifics of the system to be protected by the product being studied, and on the other hand, it determines the informativity of the test results. For example, addition of sulfur dioxide in the cell properly simulates the conditions of industrial environment. However, considerable amounts of this compound can acidify the surface electrolyte, up to modification of the metal depassivation, corrosion and inhibition mechanisms. In this case, one should not expect that VCI efficiency under natural conditions and in accelerated tests would change in a similar way. When one uses sodium chloride to simulate marine atmosphere, it should be taken into consideration that the vapor pressure of this compound is extremely low [21]. It can only be propagated in a system as an aerosol, which is created, e.g., by spraying a liquid in salt spray cabinets. However, it appears pointless to use static $\mathrm{NaCl}$ solutions as it is sometimes done in such tests.

Gas-phase tests can be carried out at room temperature [10], but it occurs more frequently that the corresponding techniques involve either permanent or intermittent heating that accelerates corrosion, ${ }^{2}$ and/or cooling of the cell or its parts that ensures vapor condensation $[2,7,22]$. The temperature variations are usually comparable with the possible daily variations and rarely exceed $35-40^{\circ} \mathrm{C}$.

Of tests under thermally controlled or similar conditions, a well proven technique was suggested by Golyanitsky. In this technique, a VCI is introduced in a solution at the cell bottom, above which corrosion specimens are placed [10]. The minimum VCI concentration that still prevents metal corrosion is determined in the tests. These tests well

\footnotetext{
${ }^{1}$ According to [1], only inhibitors with saturated vapor pressure no smaller than $10^{-6} \mathrm{mmHg}$ possess vapor-phase activity.

${ }^{2}$ The acceleration of wet corrosion due to a temperature increase by $20-30^{\circ} \mathrm{C}$ is sufficient to create corrosion damage within just a few hours.
} 
simulate the vapor-phase protection of metals by VCI solutions and process liquids on their basis. Furthermore, for many inhibitors, the values measured by this method coincide with their minimum protective concentrations in adsorbed moisture films and are among the main VCI characteristics [20].

Of the methods where corrosion acceleration is achieved by the heating of the system, the test specified by German military standard TL-8135-002 and described in [7] is popular. A steel disk-shaped specimen is mounted on a rubber stopper that seals a conical flask. Bands of a packaging material containing a VCI are also attached to it. After prolonged exposure of the metal to a VCI atmosphere, a water-glycerol solution is poured into the flask and after some time the flask is kept for $2 \mathrm{~h}$ in a cabinet at $40^{\circ} \mathrm{C}$. After the tests, the specimen is inspected and the protective capability of the material is estimated in points. Modifications of this test differing in details (composition of the corrosive electrolyte, test durations) are often recommended for acceptance testing of the quality of packing materials.

Vapor condensation, which is among the most unfavorable conditions for metal protection by VCIs and a common reason of protection failure, is simulated in gas-phase tests by creating a temperature gradient in the cells or by shock cooling.

One of the first techniques for VCI efficiency estimation under vapor condensation conditions was suggested by Vernon [22]. According to this technique, the lower part of a cell with an aqueous VCI solution on the bottom is placed in a thermostat where a temperature of $35^{\circ} \mathrm{C}$ is maintained. The top cell part where metal specimens are mounted is located outside the thermostat at ambient temperature. The method allows various VCI efficiency criteria to be used, including those characterizing complete metal protection. Obviously, the minimum protective VCI concentration obtained in this way reflects the tolerable dilution of an inhibitor by atmospheric moisture, such that the product still retains its capability for vapor-phase protection under conditions of continuous condensation of vapors on the metal. At $p_{\mathrm{VCI}}^{0}<p_{\mathrm{H}_{2} \mathrm{O}}^{0}$, complete absence of corrosion in such tests for a prolonged period of time most likely suggests that the VCI forms azeotropic mixtures with water and that the aqueous phase meets the conditions described in [17-19].

Methods in which a temperature gradient is intermittently created in a test cell are also popular.

For example, a version of the same Vernon's method involves immersing cells with VCI solutions and metal specimens in a thermostat for only 8 hours a day $[2,22]$. In the case of VCIs that are not capable of metal protection by the azeotropic mechanism, the test results will reflect the "gross" volatility of inhibitors and the protective post-effect of the surface solutions that are formed on the metal when the specimens are kept in the cell before creating a temperature gradient in it. The more volatile the inhibitor and the more irreversible its adsorption on a metal, the higher the degree of metal protection [17].

At our Institute, VCI efficiency has been estimated by the method described in $[2,19]$ for a long time. According to this method, metal specimens are mounted on a heat 
exchanger embedded in a desiccator (Fig. 1); the former is cooled with water for 3 hours a day. Either two Petri dishes, one containing water and the other a weighed portion of a VCI, or one dish containing a VCI solution are placed on the desiccator bottom. The test lasts for 45 days and is quite severe. Our experience indicates that complete suppression of steel corrosion by inhibitor solutions under these conditions is only achieved for VCIs possessing azeotropism with water or for products with $p^{0}$ close to $p_{\mathrm{H}_{2} \mathrm{O}}^{0}$ or exceeding it, so that metal cooling does not decrease the VCI concentration in the surface electrolyte.

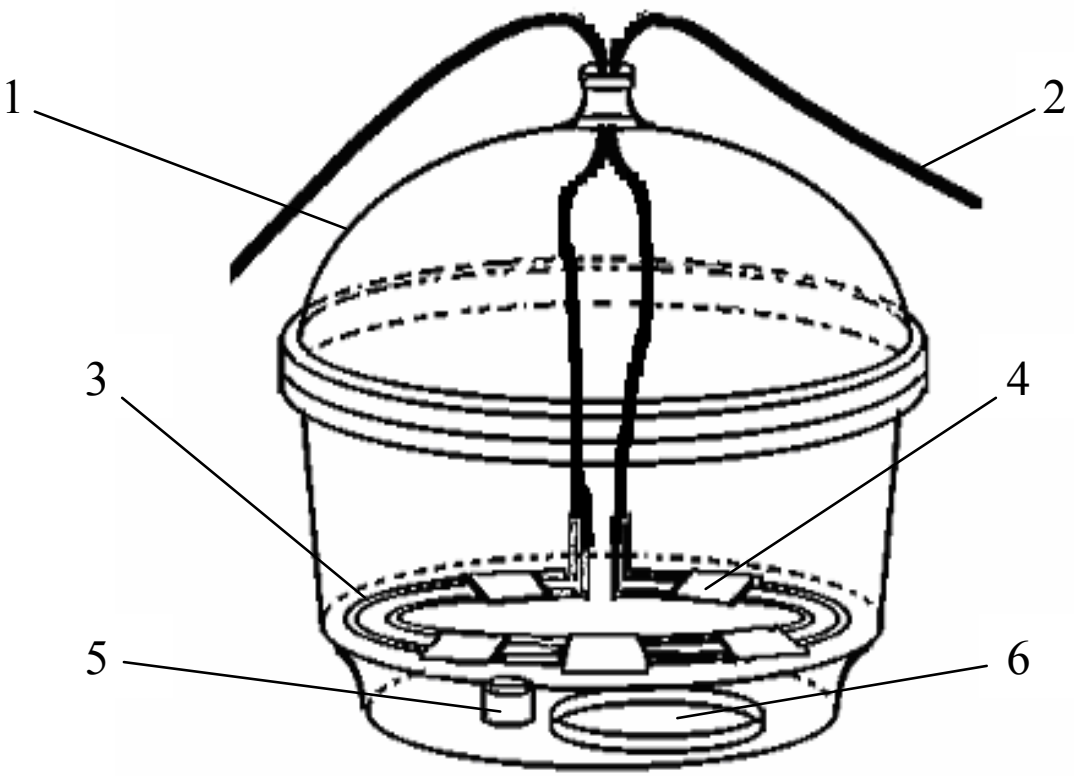

Fig. 1. Cell for testing VCI efficiency under recurrent moisture condensation conditions. 1 vacuum desiccator; 2 - rubber hose; 3 - glass heat exchanger; 4 - metal specimens; 5 - VCI; 6 - Petri dish with water.

A number of tests of this type are reported in $[2,7]$. Without dwelling on the details, let us still note that the use of water-glycerol solutions to maintain the humidity below $100 \%$ is hardly justified. A decrease in the relative humidity of the atmosphere does not change the process mechanism but just "mitigates" the condensation conditions and increases the test duration.

When the results of VCI gas-phase test results are interpreted, it should be taken into account that the results of the majority of these tests do not allow one to make unambiguous conclusions on the nature of metal protection. In fact, the corrosion of specimens can be suppressed by addition of oxygen scavengers, such as hydrazine derivatives, or desiccants decreasing the humidity below a certain level, irrespective of their volatility. Despite the external similarity, this phenomenon cannot be considered as vapor-phase corrosion inhibition. Corrosion inhibitors are compounds and combinations that, when present in a sufficient concentration in a corrosion system, can decrease the corrosion rate of metals 
without changing considerably the concentration of any corrosion agent, such as oxygen and water $[23,24]$.

\subsection{Combined tests}

A more precise conclusion on the nature of vapor-phase protection can be achieved in combined tests. They usually estimate the effect of VCIs adsorbed or condensed from a gas phase on a metal surface on the corrosion and electrochemical behavior of the metal in bulk electrolytes.

A characteristic example of combined tests is given by the method recommended for estimating the VCI efficiency in GOST (State Standard) 9.509-89 [4]. Here, metal electrodes pressed into special shells are kept for various periods of time over a VCI, then transferred into an electrochemical cell with a $\mathrm{NaCl}$ solution where potentiodynamic polarization curves are recorded.

In the combined method proposed in [5], VCIs are condensed from vapors on a cooled inert surface and washed off with a fixed volume of an electrolyte. The change in the solution corrosivity is then estimated using the linear polarization method.

The characteristics measured in these and most other combined methods reflect in an aggregate form the volatility and protective effect of an inhibitor.

In many cases, the nature of gas phase protection can also be determined on the basis of separate liquid- and gas-phase tests. Such a combination of methods is also often used for a complex estimation of the protective properties of VCI-containing materials, such as inhibited papers, polymer films, etc., that should ensure both contact and vapor-phase protection of items packed in them. Let us consider the specifics of tests for materials of this type.

\subsection{Specifics of indoor tests for inhibited packing materials}

High protective properties of materials in liquid-phase tests do not guarantee their capability for vapor-phase inhibition; likewise, efficient corrosion suppression in gas-phase tests is not always accompanied by satisfactory contact protection. A material can include non-volatile compounds that stimulate corrosion when they contact a metal but do not affect the efficiency of vapor-phase protection.

Therefore, it is common practice to use a combination of methods, e.g., the "blade" test and gas-phase tests, either those described above or others, to estimate the functional properties of inhibited packing materials. It is common to use the test from the TL-8135002 standard, or tests based on the condensation of vapors on the outer surface of a hollow metal cylinder specimen when cold water is poured therein, in this kind of combination [7]. A specimen is mounted near the strips of a test material mounted on the rubber stopper of a flask containing a water-glycerol mixture. Cooling of the metal is preceded by maintaining the cell at room temperature for a period of one day. Three hours after cooling water has been poured into a cylinder during a blank test, corrosion damage covers $100 \%$ of its 
surface. If materials with a VCI are present in the cell, the damage surface decreases, up to complete protection.

On the other hand, the barrier action contributes significantly to the protective effect of packing materials toward metal items. In fact, air-tight polyethylene packing can sometimes provide efficient metal protection in the absence of inhibitors by shielding it from exposure to moisture or other corrosive environment components [24]. Obviously, the tests described above do not reflect this capability of polymer films, papers, and composite materials on their basis.

This drawback is overcome if metal specimens are packed into the material being studied during the test. A test of this kind combining liquid- and gas-phase experiments has been developed, e.g., at our Institute to estimate the protective properties of inhibited papers and polymer films. Two methods are used to wrap or seal metal specimens into a material being studied in this test. In the first method, the surface being protected is in direct contact with the material. In the second, before packing, metal plates are placed into a shell made of laminated cardboard to prevent a contact between the specimen and the VCI carrier (Fig. 2). The packages prepared in this way are mounted in glass cells. After 5-7 days of exposure, hot water $\left(60^{\circ} \mathrm{C}\right)$ is poured onto the cell bottom. This procedure causing vapor condensation on the specimens is repeated daily. Using regular visual inspections, the time until corrosion sites appear on the specimens is determied. Estimation of the corrosion state of the specimens after a certain test time (4 months for films) is used as an additional efficiency criterion. These characteristics taken in combination make it possible to determine the effect of a VCI not only on corrosion initiation but also on corrosion propagation.

A few other tests of the type described above are mentioned elsewhere [4, 7]. Some of these imply rather complex temperature variation modes that can be maintained much more easily using cabinets.

\section{Cabinet methods}

For example, in the popular Eschke test [7], metal specimens mounted inside a bag made of inhibited film are exposed above water under cyclic temperature variation. Each four-hour cycle involves gradual heating of the system from 15 to $40^{\circ} \mathrm{C}$ followed by cooling to the initial temperature. The total test duration is 14 days.

A more complicated temperature mode of testing the protective capability of inhibited papers and films is suggested in yet another method [7] according to which metal specimens are mounted in a special shell that is packed into the material being studied. After exposure for $18 \mathrm{~h}$, water $(5 \mathrm{ml})$ is introduced into the package, and the entire package is heated from 25 to $55^{\circ} \mathrm{C}$ over a period of $3 \mathrm{~h}$. After $9 \mathrm{~h}$ of exposure at elevated temperature, the package is cooled back to $25^{\circ} \mathrm{C}$, again in 3 hours. Exposure for 9 hours at this temperature completes the cycle. During the test, either the number of cycles until corrosion damage is determined, or the condition of the samples after a certain test time is estimated. 


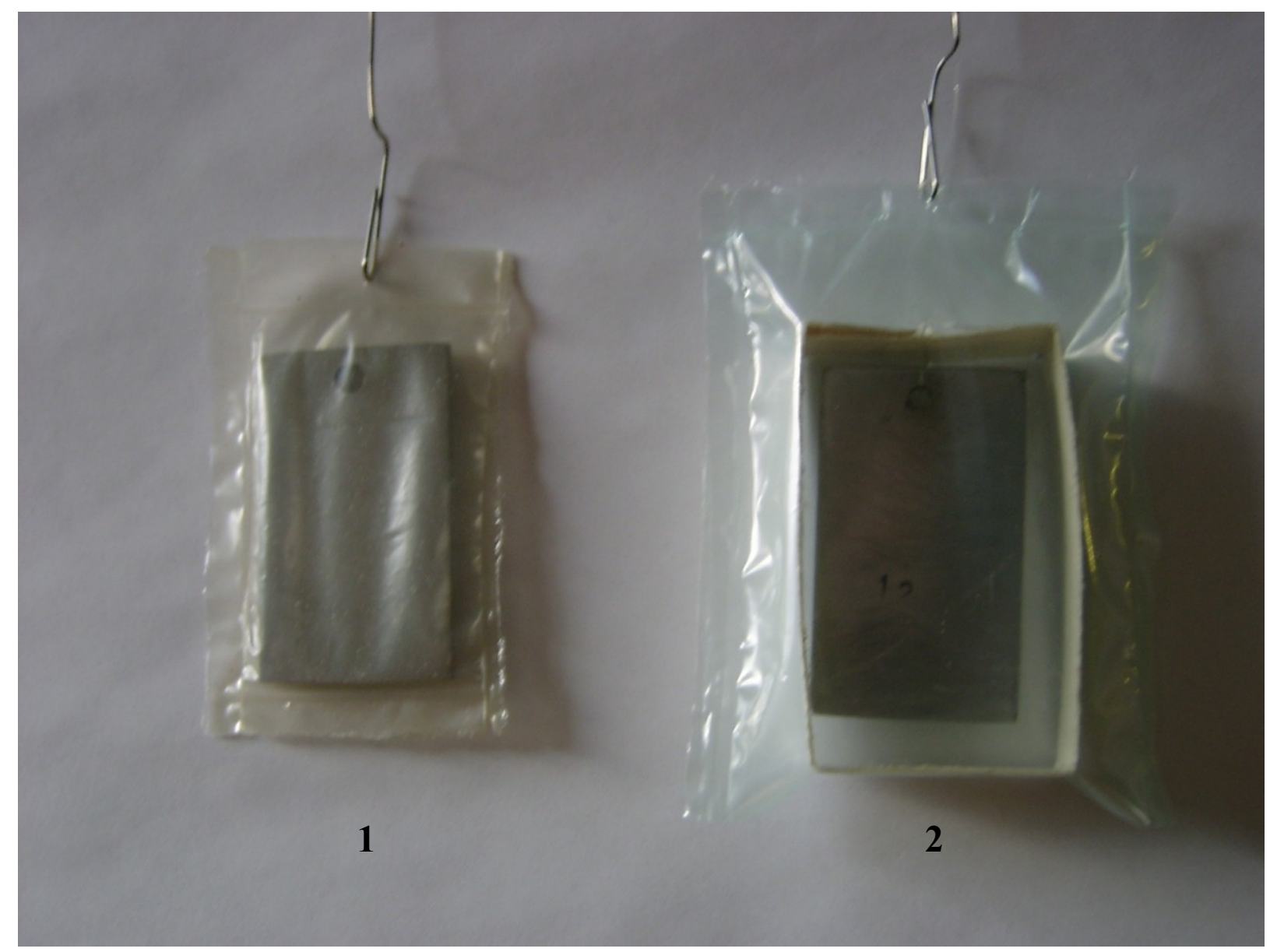

Fig. 2. Specimens packed in inhibited polymer film for estimation of the efficiency of contact protection (1) and vapor-phase protection (2).

Heating and cooling of the test cell containing $50 \mathrm{ml}$ of water and VCI or inhibited oil in the mode:

$$
25^{\circ} \mathrm{C}-10 \mathrm{~min} / 54^{\circ} \mathrm{C}-16 \mathrm{~h} / 4^{\circ} \mathrm{C}-6 \mathrm{~h} / 54^{\circ} \mathrm{C}-18 \mathrm{~h}
$$

according to the method from MIL-P-46002 [7] is also most convenient in programmable cabinets. This test is often used as a qualification test. It is considered that a material has passed this test if the specimen surface contains no more than three corrosion areas with diameters not exceeding $1 \mathrm{~mm}$ after one cycle. The drastic conditions of these tests do not allow one to use the number of cycles until occurrence of corrosion for comparative estimation of materials. It rarely exceeds two even for very efficient VCIs.

A few more cabinet testing procdeures and a system for assessment of the functional properties of temporary protection means, including VCIs, are presented in [4]. During these tests, metal specimens preserved with a VCI and packed in barrier materials and are 
successively exposed to a salt solution, elevated temperature, enhanced humidity and temperature (first without, then with moisture condensation), cold and normal atmosphere. To simulate different conditions of storage and transportation of metal items, the ratio of exposure times is slightly varied in different cabinets, but the total cycle duration remains unchanged $(76 \mathrm{~h})$. It is believed that metal protection in each cycle of these tests corresponds to six months of guaranteed metal preservation under conditions being simulated. Unfortunately, these methods are characterized by short forecast intervals (up to 2 years), as well as complex and expensive instrumentation (salt fog, heat and moisture, cold, and solar radiation cabinets), which complicates their practical application.

\section{Prospects of development of accelerated methods for VCI efficiency estimation}

Analysis of the methods described above allows one to notice that the following factors are mainly used to accelerate the tests:

- corrosive additives,

- mode of metal interaction with the corrosive electrolyte, and

- temperature mode.

Such tests are not too informative for estimation of systems in which failure of vaporphase protection is caused by mass exchange with the environment. Meanwhile, analysis of the kinetics of processes determining a decrease in VCI concentration inside a protected space provides similarity principles that are believed to be useful for VCI efficiency estimation $[13,23]$.

Simulation of VCI vaporization and water diffusion through a barrier material with thickness $l$ and surface area $S$ [23] gave the equation valid for quasi-equilibrium systems:

$$
\frac{1}{D_{\text {inh }} p_{\text {inh }}^{0}}\left[\frac{N_{\text {inh }}}{N_{\text {inh }}^{0}}-\frac{N_{\text {inh }} D_{\mathrm{H}_{2} \mathrm{O}} p_{\mathrm{H}_{2} \mathrm{O}}^{0}}{N_{\mathrm{inh}}^{0} D_{\mathrm{inh}} p_{\mathrm{inh}}^{0}}-\frac{D_{\mathrm{H}_{2} \mathrm{O}} p_{\mathrm{H}_{2} \mathrm{O}}^{0}}{D_{\mathrm{inh}} p_{\mathrm{inh}}^{0}} \ln \left(1-\frac{N_{\mathrm{inh}}}{N_{\mathrm{inh}}^{0}}\right)\right]=\frac{S t}{N_{\mathrm{inh}}^{0} R T l_{0}},
$$

where $R$ is the universal gas constant, $N_{\text {inh }}^{0}$ is the initial VCI amount, $N_{\text {inh }}$ is the VCI amount by time $t$, and $D$ represent the coefficients of diffusion through the barrier material for water or inhibitor vapor as indicated by the subscript. The $N_{\text {inh }} / N_{\text {inh }}^{0}$ ratio is functionally related to the VCI concentration in the surface water film inside the space being protected, hence relationship (1) determines the time required for VCI dilution with water in the system to a level critical for protection $\left(C_{\text {prot }}\right)$, i.e., the protection time $\left(t_{\text {prot }}\right)$, as well as the effect of the inhibitor nature, the isolating material, and the test conditions on these parameters.

It is interesting to note the structure of this equation. Its left part is a function of the VCI properties and the barrier material. The $t_{\text {prot }}$ is proportional to the value of this function, the coefficient depending on the test conditions: 


$$
f\left(D_{\mathrm{H}_{2} \mathrm{O}}, D_{\text {inh }}, C_{\text {prot }}, p_{\text {inh }}^{0}\right)=\frac{S t_{\text {prot }}}{N_{\text {inh }} R T l}
$$

This structure of the kinetics of VCI dilution with water is maintained upon changes in the model conditions over rather a broad range and provides a methodology of accelerated comparative testing of VCIs in incompletely sealed systems. ${ }^{3}$ By decreasing the VCI amount loaded initially and the barrier film thickness and increasing its surface area, an experiment under natural conditions or under conditions well simulating the former can be accelerated many-fold. The left side of dependences of type (2) will not change in this case, and the test results with the corresponding coefficients can be transferred to a real system.

\section{Conclusions}

1. Accelerated efficiency testing is an essential stage in VCI development and/or selection of optimal materials for the solution of specific practical problems. Depending on the equipment used, they are subdivided into "cabinet" and "indoor" tests. It is expedient to use characteristics that reflect the conditions of complete metal protection as VCI efficiency criteria in accelerated tests.

2. "Indoor tests" of VCIs can be subdivided into liquid-phase, gas-phase, and combined tests. They rarely replicate a real corrosion system but can simulate the onset of corrosion under various conditions of VCI usage and help in determining the base inhibitor characteristics reflecting their capability to provide vapor-phase protection.

3. A specific feature of "cabinet" tests is that they employ special cabinets that can control the temperature, humidity, and other environment characteristics according to a certain program. The goal of such tests is to simulate the most drastic conditions of storage and operation (from corrosion perspective) of a real item, or to create conditions that are even more difficult for protection and accelerate the experiment additionally.

4. The validity of estimating the functional properties of VCIs based on accelerated test results depends on the choice of the factors that accelerate corrosion. Unfavorable temperature-humidity conditions, addition of corrosive compounds to the medium, as well as decreasing the amount of the VCI added to the medium and the barrier material thickness and increasing the surface area of the latter can be used as such factors.

\footnotetext{
${ }^{3}$ Studies to determine the validity range of the regularities noted here are currently in progress at A.N.Frumkin Institute of physical chemistry and electrochemistry.
} 


\section{References}

1. I. L. Rozenfel'd and V. P. Persiantseva, Ingibitory atmosfernoi korrozii (Inhibitors of atmospheric corrosion), Moscow, Nauka, 1985 (in Russian).

2. I. L. Rozenfel'd and K. A. Zhigalova, Uskorennye metody korrozionnykh ispytanii metallov. Teoriya i praktika (Accelerated methods of metal corrosion testing. Theory and practice), Moscow, Metallurgiya, 1966 (in Russian).

3. E. M. Agres, in Fiziko-khimicheskie osnovy deistviya ingibitorov korrozii. Vypusk 2 (Physicochemical bases of action of corrosion inhibitors. Issue 2), Izhevsk, 1991, 3 (in Russian).

4. GOST (Russian State Standard) 9.509-89, Unified System of Protection from Corrosion and Ageing. Means of Temporary Protection from Corrosion. Methods for Determination of the Protective Properties, Moscow, Standards Publishing House, 1991 (in Russian).

5. E. M. Agres, Zashch. met., 1991, 27, no. 5, 681 (in Russian).

6. S. G. Tyr, O. V. Moshura and E. V. Tyr, Zashch. met., 2001, 37, no. 6, 593 (in Russian).

7. A. Furman and C. Chandler, $9^{\text {th }}$ Europ. Symp. on Corrosion Inhibitors, Ferrara, 2000, vol. 1, 493.

8. C. Fiaud, The Institute of Materials, London, 1994, 1.

9. M. N. Fokin and K. A. Zhigalova, Metody korrozionnykh ispytanii metallov (Methods of Metal Corrosion Testing), Moscow, Metallurgiya, 1986 (in Russian).

10. O. I. Golyanitsky, Letuchie ingibitory atmosfernoi korrozii metallov (Volatile inhibitors of atmospheric metal corrosion), Chelyabinsk, Chelyabinsk publishing house, 1958 (in Russian).

11. E. M. Agres and A. I. Altsybeeva, Zh. Prikl. Khim., 1993, 66, no. 10, 2217 (in Russian).

12. E. M. Agres, Zh. Prikl. Khim., 1992, 65, no. 3, 571 (in Russian).

13. N. N. Andreev and Yu. I. Kuznetsov, Int. J. Corros. Scale Inhib., 2012, 1, no. 2, 146. doi: $10.17675 / 2305-6894-2012-1-2-146-153$

14. N. N. Andreev and Yu. I. Kuznetsov, Russ. Chem. Rev., 2005, 74, 8, 685.

15. N. N. Andreev and Yu. I. Kuznetsov, in Sovremennye problemy fizicheskoi khimii (Contemporary Problems in Physical Chemistry), Moscow, "Granitsa" Publishing House, 2005, 390.

16. N. N. Andreev and Yu. I. Kuznetsov, Corrosion/04, NACE, paper no. 04413.

17. N. N. Andreev and Yu. I. Kuznetsov, Zashch. Met., 2002, 38, no. 5, 470 (in Russian).

18. N. N. Andreev and Yu. I. Kuznetsov, The European Corrosion Congress "EUROCORR 2003", Budapest, 2003, paper no. 165.

19. N. N. Andreev and Yu. I. Kuznetsov, $10^{\text {th }}$ European Symposium on Corrosion Inhibitors, Ferrara, 2005, vol. 2, 541. 
20. N. N. Andreev, Yu. I. Kuznetsov and T. V. Fedotova, Zashch. Met., 2001, 37, no. 1, 5 (in Russian).

21. Spravochnik khimika (Chemist's handbook), vol. 1, Moscow - Leningrad, 1962 (in Russian).

22. N. Stround and V. Vernon, J. Appl. Chem., 1952, 2, no. 4, 178.

23. N. N. Andreev, Razrabotka nauchnykh printsipov zashchity metallov ot korrozii organicheskimi letuchimi ingibitorami (Development of Scientific Principles of Metal Protection from Corrosion by Organic Volatile Inhibitors), Doctoral (Chem.) Dissertation, Moscow, IFKhAN, 2004 (in Russian).

24. Yu. N. Mikhailovsky, V. M. Popova and A. I. Marshakov, Zashch. Met., 2000, 36, no. 2, 546 (in Russian). 